\title{
介孔镁铝复合氧化物的成孔机理 及其结构特征
}

赵 芸 矫庆泽 D. G. Evans 段 雪**

(北京化工大学可控化学反应科学与技术基础教育部重点实验室, 北京 100029)

\begin{abstract}
摘要采用自行创制的成核/晶化隔离法, 通过调变晶化时间制备了不同粒径的层状 前体——镁铝水滑石, 并将其于 $500^{\circ} \mathrm{C}$ 焙烧, 制得了孔径分布窄、具有介孔结构特征的 镁铝复合氧化物, 且该双金属复合氧化物的晶相结构是兼有 $\mathrm{MgO}$ 晶相和层状结构的复 相结构.
\end{abstract}

\section{关键词镁铝水滑石 镁铝复合氧化物 介孔结构 晶相结构}

近年来, 以镁铝水滑石 $(\mathrm{LDH})$ 为前体制备双金属复合氧化物(LDO)备受重视, 用这种方法 制得的 LDO 微观均匀性好, 具有碱性及稳定的大比表面, 且可以通过调变前体粒子的结构来 控制其性能. 这些特点使它成为有广阔应用前景的新型碱性催化材料, 目前已广泛用于羟醛 缩合、烯烃异构化、烷基化、环氧丙烷聚合以及醇醚合成反应的催化剂 ${ }^{[1 \sim 5]}$. 醇醚合成反应中, 由于环氧化合物逐级加成, 存在分子量分布的问题，而催化剂的孔结构有可能对该反应有择 形效应. 为精确控制醇醚产物的选择性，则须制备具有一定孔径且孔径分布窄的 LDO.

$\mathrm{LDO}$ 是由前体 $\mathrm{LDH}$ 在 $500^{\circ} \mathrm{C}$ 左右焙烧形成的, $\mathrm{LDH}$ 的结构必然对 $\mathrm{LDO}$ 的晶相结构和孔 隙结构有所影响. 文中认为, LDO 的孔主要是由晶粒堆积形成的粒间孔, 前体粒子尺寸增大, 则 LDO 的孔径相应增大;前体粒子均匀, 则 LDO 的孔径分布窄化. 依据此原理, 采用本室自行 开发的成核/晶化隔离法合成尺寸均匀的 $\mathrm{LDH}$ 前体, 再经 $500^{\circ} \mathrm{C}$ 焙烧首次制备得到了孔径分布 较窄、具有介孔结构特征的 LDO. 成核/晶化隔离法的实施是采用全返混爆发式成核反应器实 现盐液与碱液的共沉淀反应, 通过控制转子的转速 $(4000 \mathrm{r} / \mathrm{min}$ )可使反应物瞬时充分接触、碰 撞, 成核反应瞬间完成, 晶核同步生长, 最大限度保证了晶化过程中晶体尺寸的均匀性. 这种 特殊方法克服了采用现有前体制备方法制备 LDO 时得到产物孔径分布宽的弊病. 若将此孔分 布窄的介孔 LDO 用于醇醚合成反应的催化剂, 则有可能得到窄分布的醇醚产物, 众所周知, 醇醚产物分子量分布的宽窄是影响产品物理和化学性能的主要因素; 产物分子量分布越宽, 愈不利于产品的合理应用. 如何获得窄分子量分布的醇醚产物一直是人们所关注的研究课题.

有关介孔 LDO 的研究, 在下述方面具有学术价值: 其合成无须模板剂、导向剂, 为介孔 材料的制备开拓了新的路线; 与以往的酸性介孔材料不同, 介孔 LDO 作为一种碱性材料 ${ }^{[6]}$, 开 拓了介孔材料新的种类; 因介孔特征, 赋予 LDO 以新的性能, 使其具有更为广阔的应用前景.

2001-03-20 收稿, 2001-08-28 收修改稿

* 中国石油化工集团公司资助项目(项目编号: X599012)

** 联系人, E-mail: duanx @ buct.edu.cn 


\section{1 实验部分}

\section{1 层状前体镁铝 LDH 的制备}

成核/晶化隔离法 ${ }^{[7]}$ :称取一定量 $\mathrm{Mg}\left(\mathrm{NO}_{3}\right)_{2} \cdot 6 \mathrm{H}_{2} \mathrm{O}(0.36 \mathrm{~mol}, 92.3 \mathrm{~g})$ 和 $\mathrm{Al}\left(\mathrm{NO}_{3}\right)_{3} \cdot 9 \mathrm{H}_{2} \mathrm{O}$ $(0.12 \mathrm{~mol}, 45.0 \mathrm{~g})$ 溶于去离子水中配成混合盐溶液, 另称取一定量 $\mathrm{NaOH}(0.77 \mathrm{~mol}, 30.9 \mathrm{~g})$ 和 $\mathrm{Na}_{2} \mathrm{CO}_{3}(0.24 \mathrm{~mol}, 25.4 \mathrm{~g})$ 溶于去离子水中配成混合碱溶液, 将 2 种溶液迅速于全返混爆发式成 核反应器中混合，剧烈循环搅拌 $1 \mathrm{~min}$, 后将浆液于 $100^{\circ} \mathrm{C}$ 下回流晶化一定时间，过滤，洗涤， 干燥, 得镁铝 LDH.

\subsection{LDO 的制备}

将前体 LDH 置于马福炉中于 $500^{\circ} \mathrm{C}$ 焙烧 $3 \mathrm{~h}$ ，即得 LDO.

\subsection{LDH 和 LDO 的表征}

在 D5005D X 射线衍射仪上测定 LDH 和 LDO 的 XRD 图, 表征其晶体结构, 根据(003)和 (110)衍射峰之半峰宽推测 LDH 初级粒子尺寸. 主要操作条件: $40 \mathrm{kV}, 20 \mathrm{~mA}, \mathrm{Cu}$ 靶.

用日立 H-800 透射电子显微镜表征 LDO 晶体形貌.

在 Sorptomatic1990 吸脱附仪上, 用低温 $\mathrm{N}_{2}$ 吸附法表征 LDO 的孔隙结构, 脱气温度 $300^{\circ} \mathrm{C}, \mathrm{BET}$ 法计算比表面, 脱附分支计算孔径分布.

在 Micromeritics Auto Pore III (WIN9400)仪器上, 用压永法分析测定 LDO 的孔隙结构.

\section{2 结果与讨论}

\subsection{LDO 成孔机理分析}

$\mathrm{LDH}$ 在 $500^{\circ} \mathrm{C}$ 左右焙烧后, 于空气中或水和二氧化碳存在的条件下，又能恢复其原有结 构, 这种结构记忆效应表明 LDH 在焙烧过程中, 层状结构可能未完全破坏, 只是有序度稍有 下降，同时也说明前体结构对 LDO 的结构会有影响. 因此控制前体 LDH 的结构可望实现 LDO 的结构控制.

对 LDH 而言, 其初级粒子尺寸较小, 在表面能作用下会聚集在一起形成次级粒子, 这样, 焙烧后初级粒子间的空隙构成了 LDO 的孔系统. 孔系统中孔的形状和大小与初级粒子的形状 和大小有关，不同尺寸的初级粒子聚集形成的粒间孔不同，初级粒子尺寸越大，其堆积形成的 粒间孔也应越大. 前体粒子均匀, 则 LDO 的孔径分布窄化. 用成核/晶化隔离法制备 LDH，晶 核的形成是在盐溶液和碱溶液共混的瞬间完成, 且回流过程中剧烈摚拌消除了溶液浓度梯度 对晶体生长的影响, 在恒定温度下影响晶体结构的因素为晶化时间, 随晶化时间延长, 前体 LDH 晶粒尺寸应增大，且其晶粒尺寸分布应窄化. 所以有望通过控制前体晶化时间，来控制 前体粒子尺寸及分布，从而实现 LDO 孔隙结构的精准控制.

\subsection{1 不同晶化时间前体 LDH 的晶体结构和晶粒尺寸 图 1 为用成核/晶化隔离法制备的不} 同晶化时间 LDH 的 XRD 图, 可以看出, 晶化 2, 6, 13 h 的产物均具有典型的 LDH 晶体特征衍 射峰, 且谱图基线低, 晶体结构完整. 从表 1 可看出样品 $d_{003}, d_{006}, d_{009}$ 各衍射峰的 $d$ 值间均存 在良好的倍数关系, 表明样品具有较为理想的层状结构.

$\mathrm{LDH}$ 晶体为六方晶系, 根据衍射峰指标化和 $d$ 值, 可计算出晶胞参数 $a$ 和 $c$, 参数 $a$ 为相 邻六方晶胞中金属原子间的距离, 参数 $c$ 为晶胞的厚度 ${ }^{[8]}$. 表 1 列出了晶胞参数的计算结果, 


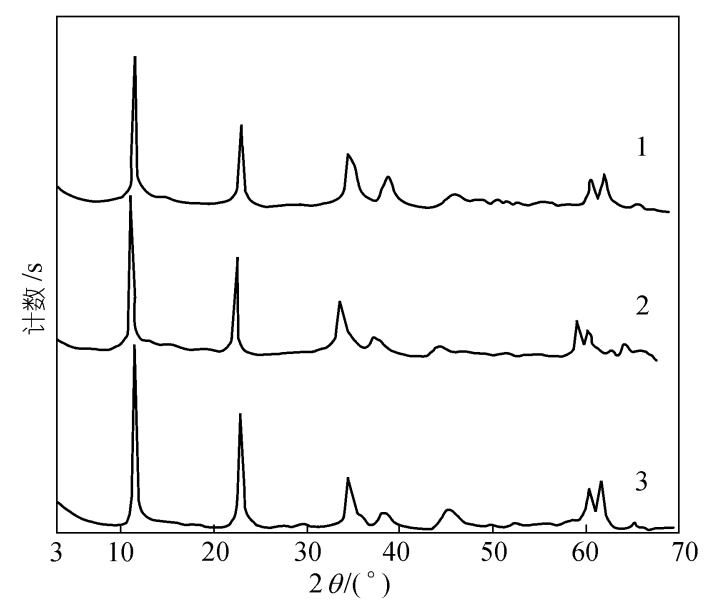

图 1 不同晶化时间 LDH 的 XRD 图 1 示晶化 $2 \mathrm{~h} ; 2$ 示晶化 $6 \mathrm{~h} ; 3$ 示晶化 $13 \mathrm{~h}$
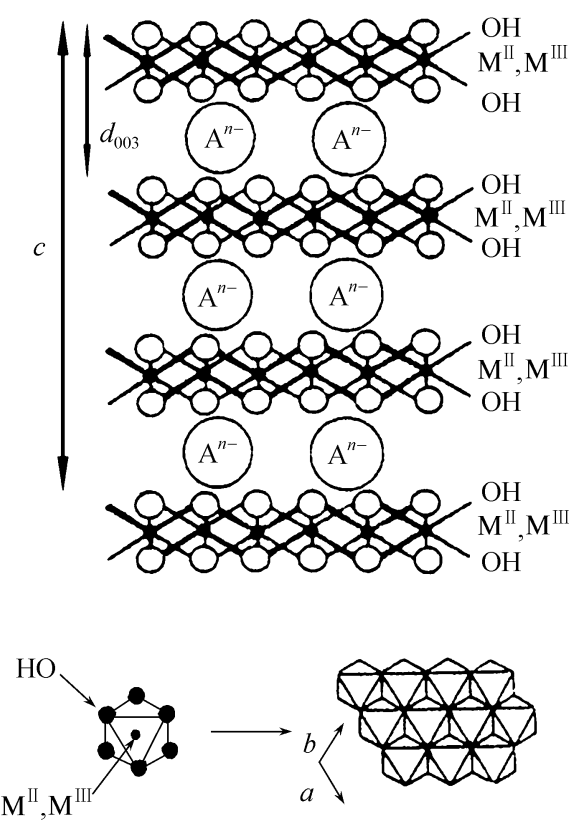

图 $2 \mathrm{LDH}$ 的结构示意图 的 3 倍, 表明每个晶胞是由 3 层层板组成, 图 2 为 $\mathrm{LDH}$ 的晶体结构示意图 ${ }^{[9]}$.

从图 1 还可看出, 随晶化时间延长, LDH 特征峰明显增强且尖锐, 对比(003)及(110)衍射峰 之半峰宽的数值(见表 1) 可知, 晶化时间延长, (003)及(110)衍射峰的半峰宽明显降低, 表明随 晶化时间延长, LDH 结晶度增加, 晶粒尺寸增大. 根据 Scherrer 公式:

$$
D_{h k l}=R \lambda / \beta \cos \theta,
$$

式中 $D_{h k l}$ 为沿晶面垂直方向的晶粒尺寸; $R$ 为 $\operatorname{Scherrer}$ 常数 $(0.89) ; \lambda$ 为人射线波长 $(0.1542 \mathrm{~nm}) ; \theta$ 为 Bragg 角; $\beta$ 为衍射峰的半峰宽.

可推测水滑石的晶粒尺寸 ${ }^{[10]}$. 从表 1 中可知晶化 $2 \mathrm{~h}$ 的样品的晶粒尺寸沿 $c$ 轴方向约 22 $\mathrm{nm}$, 估算由 28 层层板组成, 沿 $a$ 轴方向约 $24 \mathrm{~nm}$; 晶化时间为 $13 \mathrm{~h}$ 的样品晶粒尺寸沿 $c$ 轴方

表 1 不同晶化时间前体 LDH 的 XRD 结构参数

\begin{tabular}{llll}
\hline 晶化时间 $/ \mathrm{h}$ & 2 & 6 & 13 \\
\hline$d_{003} / \mathrm{nm}$ & 0.779 & 0.780 & 0.778 \\
$d_{006} / \mathrm{nm}$ & 0.389 & 0.389 & 0.388 \\
$d_{009} / \mathrm{nm}$ & 0.260 & 0.261 & 0.261 \\
$d_{110} / \mathrm{nm}$ & 0.153 & 0.153 & 0.153 \\
$(003)$ 之 $\left.\mathrm{W}_{1 / 2} /{ }^{\circ}\right)$ & 0.360 & 0.334 & 0.309 \\
$(110)$ 之 $\mathrm{W}_{1 / 2} /\left(^{\circ}\right)$ & 0.386 & 0.319 & 0.285 \\
晶胞参数 $a$ & 0.306 & 0.306 & 0.306 \\
晶胞参数 $c$ & 2.336 & 2.340 & 2.334 \\
$a$ 轴方向晶粒尺寸 ${ }^{\mathrm{a})} / \mathrm{nm}$ & 23.6 & 28.5 & 31.9 \\
$c$ 轴方向晶粒尺寸 $\mathrm{a}) / \mathrm{nm}$ & 21.9 & 23.6 & 25.5 \\
晶胞堆垛层数 & 28 & 30 & 33 \\
\hline
\end{tabular}

a) Scherrer 公式的计算值 
向约 $26 \mathrm{~nm}$, 估算由 33 层层板组成, 沿 $a$ 轴方向约 $32 \mathrm{~nm}$. 可见, 随晶化时间延长, 晶粒沿 $c$ 轴方向和 $a$ 轴方向尺寸都增大, 但晶粒沿 $a$ 轴方向生长得快, 这是因为(110)晶面其面网密度 小, 晶面间距也小, 从而相邻的面网之间的引力就大, 因此优先生长. 由此可知, 通过调变晶 化时间可有效控制 LDH 晶粒尺寸.

2.1.2 LDO 的孔隙结构 将上述晶化时间分别为 $2,6,13 \mathrm{~h}$ 的 $\mathrm{LDH}$ 样品于 $500^{\circ} \mathrm{C}$ 焙烧后, 进 行孔隙结构表征, 结果如图 3 和表 2. 可看出, 随前体晶化时间延长, 孔径分布变窄, 且孔径向 大的方向移动, 最可几孔径从 $24.1 \mathrm{~nm}$ 增大至 $28.7 \mathrm{~nm}$, 比表面积增大. 由前述可知, 晶化时间 延长, 前体 $\mathrm{LDH}$ 晶粒尺寸增大. 一般随晶粒尺寸增大, 单位重量的比表面变小, 但此处相反, 比表面随晶粒尺寸增大而稍有增大，这是因 LDO 的比表面不仅与前体晶粒尺寸有关，还与 前体的结晶度有关, 无定形部分会影响焙烧产物的比表面，使比表面降低. 如前所述，随晶 化时间延长, $\mathrm{LDH}$ 晶粒尺寸增大的同时结晶度也在提高, 这样晶粒尺寸的增大和无定形部分 的减少两者综合作用的结果使 LDO 的比表面略有增大. 可见，随前体晶化时间延长，前体 LDH 晶粒尺寸增大, 焙烧后 LDO 孔径相应增大. 可以得出结论:控制前体晶化时间, 可控制前 体粒子尺寸, 从而控制焙烧产物的孔结构.
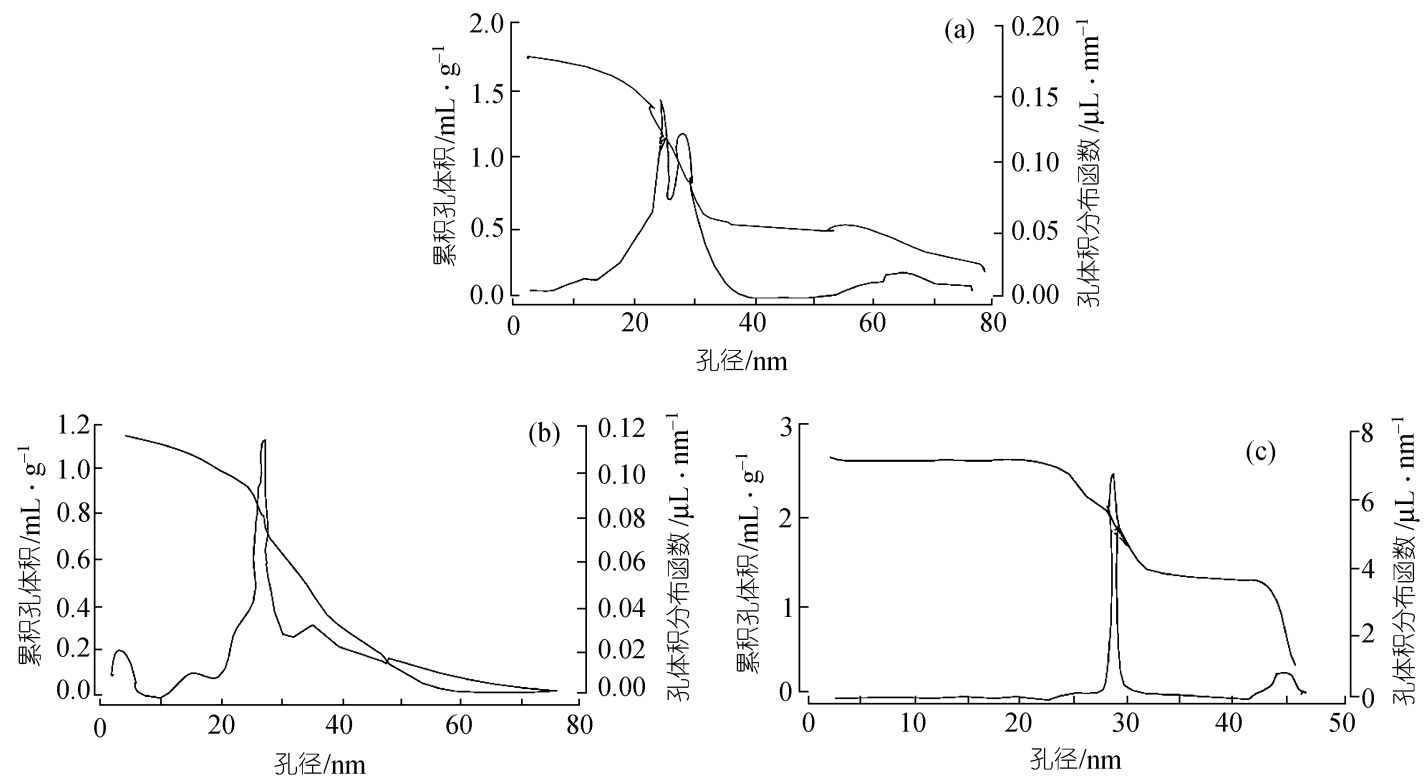

图 3 不同前体晶化时间 LDO 的孔径分布图

(a) 前体晶化 $2 \mathrm{~h}$; (b) 前体晶化 $6 \mathrm{~h}$; (c) 前体晶化 $13 \mathrm{~h}$

表 2 不同前体晶化时间 LDO 的孔结构数据

\begin{tabular}{|c|c|c|c|c|c|c|}
\hline \multirow{2}{*}{ 前体晶化时间/h } & \multicolumn{3}{|c|}{ 孔径分布 $/ \%$} & \multirow{2}{*}{ 最可几孔径/nm } & \multirow{2}{*}{ 比表面 $\mathrm{m}^{2} / \mathrm{g}$} & \multirow{2}{*}{ 孔容 $\mathrm{cm}^{3} / \mathrm{g}$} \\
\hline & $0 \sim 10 \mathrm{~nm}$ & $10 \sim 20 \mathrm{~nm}$ & $>20 \mathrm{~nm}$ & & & \\
\hline 2 & 2.3 & 11.1 & 86.6 & 24.1 & 179.5 & 1.11 \\
\hline 6 & 4.7 & 7.2 & 88.1 & 26.3 & 183.8 & 0.83 \\
\hline 13 & 0.2 & 0.2 & 99.6 & 28.7 & 192.4 & 1.13 \\
\hline
\end{tabular}


图 3 中前体晶化 $2 \mathrm{~h}$ 的 LDO 孔分布宽, 这是由于前体 LDH 中有多种尺寸的初级粒子. 众 所周知, 晶体生长过程中小颗粒溶解、大颗粒长大, 成核/晶化隔离法制备 LDH, 成核在瞬间 完成, 晶化 $2 \mathrm{~h}$ 时, 处在晶体生长的过程中, 晶粒尺寸均匀性相对较差, 这就导致体系中晶粒 尺寸分布宽，随晶化时间延长，晶粒趋于均匀，因而焙烧产物 LDO 的孔径趋于均匀.

图 3(c) 表明前体晶化 13h 的 LDO 孔径 分布窄, 具有明显的介孔结构特征: 最可几孔 径为 $28.7 \mathrm{~nm}$, 比表面为 $192.4 \mathrm{~m}^{2} / \mathrm{g}$, 孔容为 $1.13 \mathrm{~cm}^{3} / \mathrm{g}$. 对该介孔 LDO, 用压录法测定孔 径分布来验证其孔隙结构特征, 结果与低温 氮吸附法一致, 也表明该样品孔结构规整, 孔径均一, 具有介孔特征(见图 4), 有较大比 表面积 $204.1 \mathrm{~m}^{2} / \mathrm{g}$ 和孔容 $2.20 \mathrm{~cm}^{3} / \mathrm{g}$. 但压永 法测得的最可几孔径约为 $40 \mathrm{~nm}$, 与低温氮吸 附法的 $28.7 \mathrm{~nm}$ 相差较大, 孔容也有较大差别, 这可能是由于两种方法本身的差别造成的, 压录法测大孔更为准确.

\section{2 介孔 LDO 的晶相结构}

依据 2.1 节中的成孔机理，控制合适的前

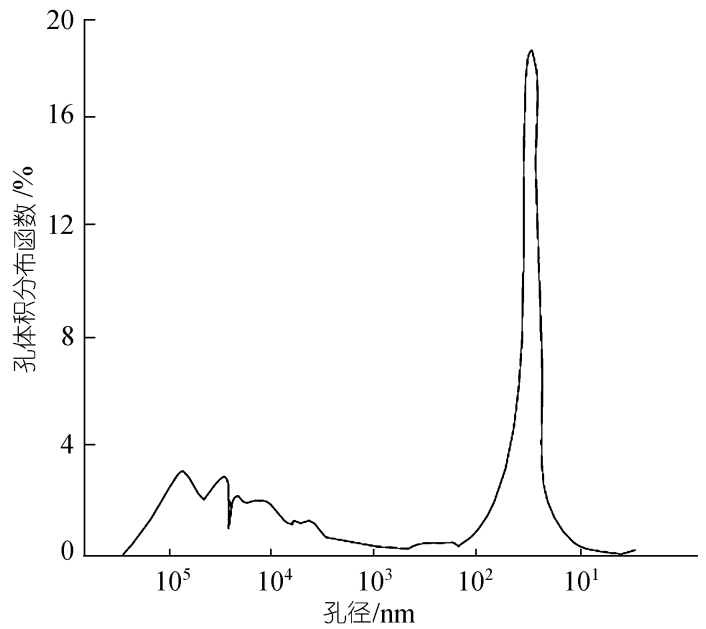

图 4 介孔 LDO 的孔径分布图(压永法) 体晶化时间制备得到了孔径均匀、具有介孔结构特征的 LDO, 并对其晶相结构进行了表征.

对比介孔 $\mathrm{LDO}$ 与前体 $\mathrm{LDH}$ 和氧化镁的 XRD 图(见图 5), 可看出 LDO 的 XRD 图上没有 $\mathrm{LDH}$ 晶体的特征衍射峰, 其特征衍射峰基本与 $\mathrm{MgO}$ 一致, 多了一个 $d=0.253 \mathrm{~nm}$ 的反射, 峰

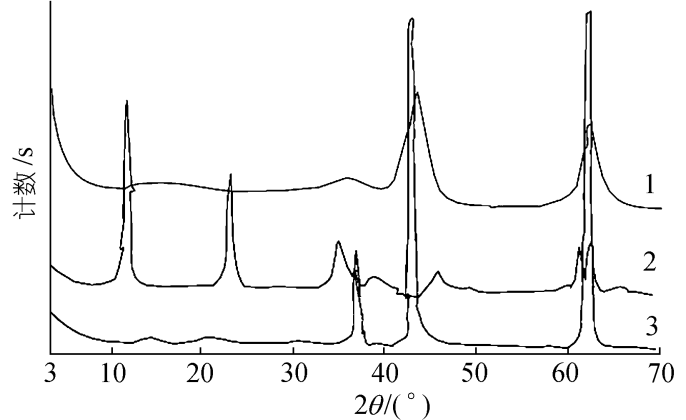

图 5 介孔 LDO 的 XRD 图 1, LDO; 2, LDH; $3, \mathrm{MgO}$ 形不如 $\mathrm{MgO}$ 的尖锐, 说明 LDO 的晶体结构不如 $\mathrm{MgO}$ 完整. 根据文献 $[11,12], d=0.253 \mathrm{~nm}$ 的反 射反映了 $\mathrm{MgO}$ 晶格内部阳离子四面体结构的形 成，也就是说层状前体 LDH 在高温焙烧过程中， 一部分阳离子从八面体迁移到四面体位置, 这 种处于四面体位置的阳离子可能属于含有过量 镁的非化学计量的尖晶石相.

LDO 的 TEM 照片(见图 6)显示出其仍然具 有明显的层状结构特征, 这一结果表明: 在本实 验条件下, 前体 LDH 在高温焙烧过程中层板结 构不会崩塌, 这与文献[13]结果一致.

由上述结果可知, $\mathrm{LDO}$ 既具有 $\mathrm{MgO}$ 的晶相结构, 又保持前体 $\mathrm{LDH}$ 的层状结构, 因此我们 设想 $\mathrm{LDO}$ 是一种复相结构——兼有 $\mathrm{MgO}$ 晶相和层状结构的复相结构.

\section{3 影响 LDO 介孔结构的因素分析}

从 LDO 的制备及成孔机理, 可以推断, 影响 LDO 介孔结构的因素主要为前体 LDH 结构 及焙烧温度.

前者包括前体 LDH 的晶体结构和前体粒子尺寸及其均匀性. 其中前体 LDH 的晶体结构 


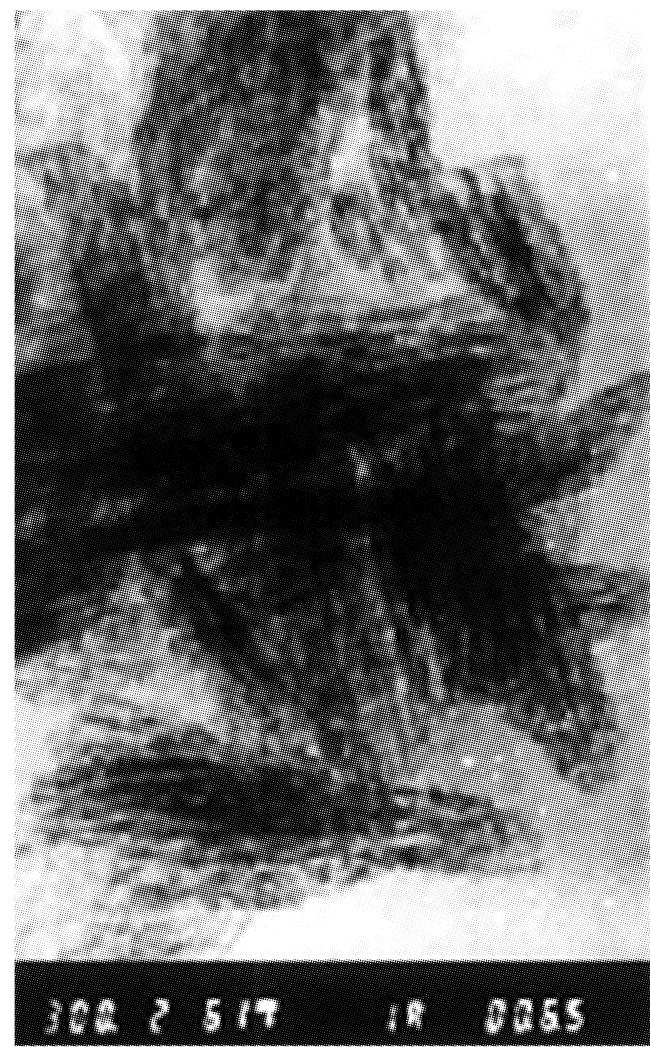

图 6 介孔 LDO 的 TEM 照片(放大 300000 倍)

影响 LDO 的晶相结构, 前体粒子尺寸影响 LDO 的孔径大小, 前体粒子的均匀性影响 LDO 的孔径分布. 而所有这些因素都受前体 LDH 合成条件的影响，如晶化温度、晶化时间、 投料镁铝比等 ${ }^{1), 2 \text {. }}$.

焙烧温度对 LDO 孔隙结构的影响也很大. 根据本室多年的研究以及其他文献资料, 对 前体 $\mathrm{LDH}, 450 \sim 500^{\circ} \mathrm{C}$ 是形成 $\mathrm{LDO}$ 最合适的焙 烧温度, 焙烧温度低于此范围, LDH 分解不完 全, 未完全形成 LDO 的结构; 焙烧温度过高, 前体 LDH 在焙烧过程中容易烧结, 出现尖晶 石相, 导致比表面降低 ${ }^{[6]}$ 及孔结构的变化. 将 前述晶化 $13 \mathrm{~h}$ 的前体 $\mathrm{LDH}$ 于 $700^{\circ} \mathrm{C}$ 焙烧, 其焙 烧产物的孔径分布为：0 10 nm, 2.4\%; 10 20 $\mathrm{nm}, 3.6 \%$; $>20 \mathrm{~nm}, 94.0 \%$; 比表面为 $160.7 \mathrm{~m}^{2} / \mathrm{g}$, 明显比 $500^{\circ} \mathrm{C}$ 焙烧得到的 LDO 孔分布宽, 比表 面低, 表明焙烧温度确实对 LDO 孔隙结构有 影响.

\section{3 结论}

(i) 在无模板剂、无导向剂的条件下, 制 备得到了碱性介孔材料 LDO. 其孔径分布窄, 最可几孔径为 $28.7 \mathrm{~nm}$, 比表面 $192.4 \mathrm{~m}^{2} / \mathrm{g}$, 孔容 $1.13 \mathrm{~cm}^{3} / \mathrm{g}$.

(ii) 在本实验条件下制备的介孔 LDO, 其晶相结构兼具氧化镁晶相和层状结构.

(iii) 控制层状前体 LDH 的晶粒尺寸, 可有效控制 LDO 的孔径尺寸及分布.

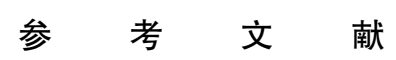

1 Cavani F, Trifiro F, Vaccari A. Hydrotalcite-type anionic clays: Preparation, properties and applications. Catalysis Today, 1991, 11: 173 301

2 张亦勍, 杜以波, 段 雪, 等. 窄分布醇醚合成用催化剂的篮选与催化作用分析. 精细化工, 1999, 16(4): 35 39

3 Ivereva M V, Makarov M G, Kapustin A E. Reaction of alcohols with $\alpha$-oxides in the presence of layered double catalysts. React Kinet Catal Lett, 1998, 65(2): 349 354

4 Rao K K, Gravelle M, Valente J S, et al. Activation of Mg-Al hydrotalcite catalysts for aldol condensation reactions. J Catal, 1998, 173: 115 121

5 Kohjiya S, Sato T, Nakayama T, et al. Polymerization of propylene oxide by calcined synthetic hydrotalcite. Makromol Chem, Rapid Commun, 1981, 2: 231 233

1) 张亦勍. 窄分布醇醚合成中固体碱催化材料的结构与性能. 北京化工大学硕士研究生学位论文, 1999. 22 36

2) 张春英. 层状 LDHs 新型制备方法的创制及其煅烧产物的紫外阻隔性能研究. 北京化工大学硕士研究生学位论文, 2000. 21 28 
6 Mckenzie A L, Fishel C T, Davis R J. Investigation of the surface structure and basic properties of calcined hydrotalcites. J Catal, 1992, 138: 547 561

7 段 雪, 矫庆泽, 李 蕾. CN Patent, 99119385. 7 1999-9-14

8 Han S H, Zhang C G, Hou W G, et al. Study on the preparation and structure of positive sol composed of mixed metal hydroxide. Colloid Polym Sci, 1996, 274: 860 865

9 Constantino V R L, Pinnavaia T J. Basic properties of $\mathrm{Mg}_{1-x}^{2+} \mathrm{Al}_{x}^{3+}$ layered double hydroxides intercalated by carbonate, hydroxide, chloride, and sulfate anions. Inorg Chem, 1995, 34: 883 892

10 苏延否, 侯万国, 孙德军, 等. Mg-Fe-LDHs 纳米颗粒的合成及其阴离子交换容量的研究. 高等学校化学学报, 1999, 20: $1012 \sim 1016$

11 Bellotto M, Rebours B, Clause O, et al. Hydrotalcite decomposition mechanism: A clue to the structure and reactivity of spinel-like mixed oxides. J Phys Chem, 1996, 100: 8535 8542

12 Rebours B, Caillerie J B, Clause O. Decoration of nickel and magnesium oxide crystallites with spinel-type phases. J Am Chem Soc, 1994, 116: 1707 1717

13 Reichle W T, Kang S Y, Everhardt D S. The nature of the thermal decomposition of a catalytically active anionic clay mineral. J Catal, 1986, 101: 352 359 